\title{
Corneodesmosin Expression in Psoriasis Vulgaris Differs from Normal Skin and Other Inflammatory Skin Disorders
}

\author{
Michael Allen, Akemi Ishida-Yamamoto, John McGrath, Simon Davison, \\ Hajime lizuka, Michel Simon, Marina Guerrin, Adrian Hayday, Robert Vaughan, \\ Guy Serre, Richard Trembath, and Jonathan Barker
}

St. John's Institute of Dermatology (MA, JM, SD, JB), Immunobiology (AH), and Tissue Typing (RV), King's College, London, and Division of Medical Genetics (RT), University of Leicester, Leicester, United Kingdom; Department of Dermatology (Al-Y, HI), Asahikawa Medical College, Asahikawa, Japan; and Biology and Pathology of the Cell (MS, MG, GS), INSERM CJF 9602-IFR 30, University of Toulouse, Toulouse, France

\begin{abstract}
SUMMARY: Corneodesmosin (Cdsn) is a late differentiation epidermal glycoprotein putatively involved in keratinocyte adhesion. The Cdsn gene lies within the susceptibility region on chromosome 6p21.3 (PSORS1) for psoriasis, a common chronic disfiguring skin disease. A particular allelic variant of Cdsn has a strong association with psoriasis. Therefore, genetically and biologically, Cdsn is a possible candidate gene for psoriasis susceptibility. To investigate a potential role for Cdsn in psoriasis pathogenesis, protein expression studies were performed by quantitative immunohistochemistry on normal skin, psoriatic skin (lesional and nonlesional), and other skin disorders using monoclonal antibodies (G36-19 and F28-27). In normal and nonlesional skin, Cdsn was expressed in stratum corneum and one or two layers of superficial stratum granulosum. In lesional psoriasis, there was a significant increase in Cdsn expression, which was observed in multiple layers of stratum spinosum and in stratum corneum. The expression pattern varied from granular, cytoplasmic immunoreactivity to cell surface labeling with weakly immunoreactive cytoplasm. In chronic atopic dermatitis, lichen planus, mycosis fungoides, and pityriasis rubra pilaris, Cdsn immunoreactivity was confined to stratum corneum and upper stratum granulosum with no stratum spinosum immunoreactivity. Immunoelectron microscopy of normal and lesional psoriatic skin demonstrated Cdsn release concomitant with involucrin incorporation into cell envelopes and completed before mature envelope formation. Extracellular release of Cdsn occurred at a lower level of the epidermis in psoriasis than normal skin. These protein expression studies provide evidence of altered Cdsn expression in psoriasis consistent with a role of Cdsn in disease pathogenesis. Further functional and genetic studies of Cdsn are justified to determine its role as a potential psoriasis-susceptibility factor. (Lab Invest 2001, 81:969-976).
\end{abstract}

C orneodesmosin (Cdsn) is a late differentiation epidermal glycoprotein expressed in the stratum granulosum and stratum corneum of normal skin and thought to function as a keratinocyte adhesion molecule (Serre et al, 1991). Cdsn mRNA expression is restricted to keratinocytes of the granular layer in normal human epidermis (Zhou and Chaplin, 1993). In the stratum granulosum of normal skin, Cdsn is present as a 52 to $56 \mathrm{kd}$ glycoprotein, whereas in the stratum corneum it exists as smaller macromolecules of 30 to $45 \mathrm{kd}$ (Simon et al, 1997). Cdsn is initially observed intracellularly within keratinosomes and is then translocated to the cell surface, where it is incorporated into desmosomes, modifying them to

Received February 28, 2001.

This study was funded by the National Psoriasis Foundation USA (MHA), the Ministry of Education, Science, Sports and Culture of Japan (AI-Y), the Ministry of Health and Welfare, Japan (HI), and the Wellcome Trust (JNWNB and RCT).

Address reprint requests to: Professor Jonathan N. W. N. Barker, St. John's Institute of Dermatology, St. Thomas's Hospital, Lambeth Palace Road, London SE1 7EH, UK. E-mail: jonathan.barker@kcl.ac.uk corneodesmosomes (Serre et al, 1991). Some desmosomal proteins are important in maintaining keratinocyte cell-cell adhesion, and it is likely that Cdsn forms part of the adhesive network (Serre et al, 1991). Cdsn has a high glycine content, which implies a potential to form adhesive loop structures at the cell surface, as seen with other epidermal proteins (Guerrin et al, 1998). Progressive proteolytic digestion of Cdsn has been proposed, which would account for the decrease in molecular size between the stratum granulosum and stratum corneum, and the gradual reduction and eventual loss of adhesive function (Simon et al, 1997) with associated scale formation.

Psoriasis is a common, chronic, disfiguring skin disease characterized by epidermal proliferation and a loss of keratinocyte differentiation, with vascular changes and accumulation of inflammatory cells (Camp, 1998). Current evidence indicates an essential role for $\mathrm{T}$ cells in the disease pathogenesis (Barker, 1991; Nickoloff and Wrone-Smith, 1999). Furthermore, it is clear that these pathophysiologic events take place on an important genetic background (Elder et al, 1994). Multiple studies indicate genetic linkage of 
psoriasis to a locus on chromosome $6 \mathrm{p} 21.3$ (PSORS1), accounting for $35 \%$ to $50 \%$ of the genetic contribution to psoriasis (Burden et al, 1998; Nair et al, 1997; Trembath et al, 1997). Linkage disequilibrium mapping studies have refined the PSORS1 locus to an approximately $300 \mathrm{~kb}$ region of chromosome $6 \mathrm{p} 21.3$ (Balendran et al, 1999; Nair et al, 2000; Oka et al, 1999), which contains the genes for HLA-C and Cdsn (160 kb telomeric of HLA-C) (Zhou and Chaplin, 1993).

In addition to increasing genetic evidence (Nair et al, 2000; Oka et al, 1999) against HLA-C as a susceptibility gene for psoriasis, the biological characteristics of HLA-C do not support a functional role in psoriasis. However, the major histocompatibility complex contains many non-HLA genes and HLA-C may simply be acting as a genetic marker for the psoriasissusceptibility gene(s) in close linkage disequilibrium with HLA-C. One such gene is the Cdsn gene, which, because of its genomic position and biological function, is a good potential candidate for psoriasis susceptibility. Strong association has been demonstrated between alleles of the Cdsn gene and psoriasis (Allen et al, 1999; Jenisch et al, 1999; Tazi Ahnini et al, 1999). A characteristic feature of psoriasis is hyperkeratosis and increased shedding of epidermal scale, suggesting a possible abnormality of keratinocyte adhesion. Thus, both genetic and functional data indicate a potential important role for Cdsn in psoriasis pathogenesis.

To further investigate the role of Cdsn in psoriasis pathogenesis, we performed protein expression studies at both histologic and ultrastructural levels using monoclonal antibodies specific for conserved epitopes of the Cdsn molecule. Expression patterns of Cdsn in psoriasis were compared with expression patterns in normal skin and in other skin conditions to identify disease-specific components of Cdsn expression unique to psoriasis.

\section{Results}

\section{Immunoreactivity}

Quantification. Throughout this study, identical immunoreactivity results were obtained on frozen and paraffin-embedded tissue. A distinct difference was observed in the immunoreactivity pattern of normal skin compared with psoriatic skin. This difference was confirmed by the two-sample $t$ test, which showed a statistically significant increase in the number of living layers of the epidermis in psoriasis expressing Cdsn (mean, 6.3) compared with expression of Cdsn in normal skin (mean, 1.9, $p<0.01$ ) (Fig. 1). A statistically significant increase $(p<0.01)$ was also demonstrated in the number of living cell layers expressing Cdsn in lesional psoriatic skin (mean, 5.8) compared with nonlesional psoriatic skin (mean, 1.9) (Fig. 1). No statistically significant differences were observed in the numbers of living layers expressing Cdsn in normal skin and nonlesional psoriatic skin.

Normal Skin. In both frozen and paraffin-embedded normal skin, Cdsn was expressed in a maximum of two cell layers of the upper stratum granulosum and less intensely throughout the full thickness of the stratum corneum. There was no Cdsn immunoreactivity in the stratum spinosum, although acrosyringia were also immunoreactive for Cdsn. Stratum granulosum immunoreactivity was cytoplasmic, with a cell surface component to the immunoreactivity appearing in some of the flattened nucleated cells in the lower stratum corneum (Fig. 2, a and b). Cdsn immunoreactivity was retained on the surface of corneocytes within the stratum corneum.

Psoriasis. In contrast to the above observations, in frozen and paraffin-embedded sections of lesional psoriasis, G36-19 and F28-27 antibodies were immunoreactive in multiple layers of epidermis extending

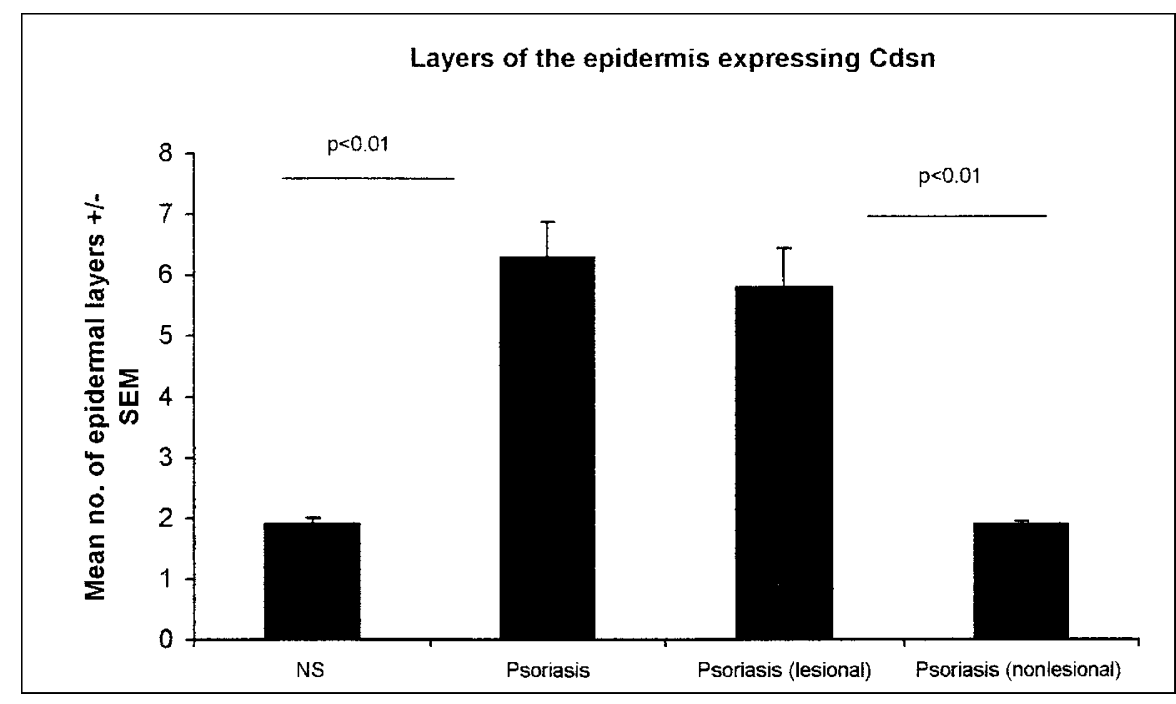

Figure 1.

Comparison of corneodesmosin (Cdsn) expression in the epidermis of normal skin (NS) and psoriasis. There is a statistically significant increase in Cdsn expression in psoriatic epidermis compared with normal epidermis. In paired skin biopsies $(n=17)$ of lesional and nonlesional skin from psoriatic individuals, there is also a statistically significant increase in Cdsn expression in lesional psoriatic epidermis. 

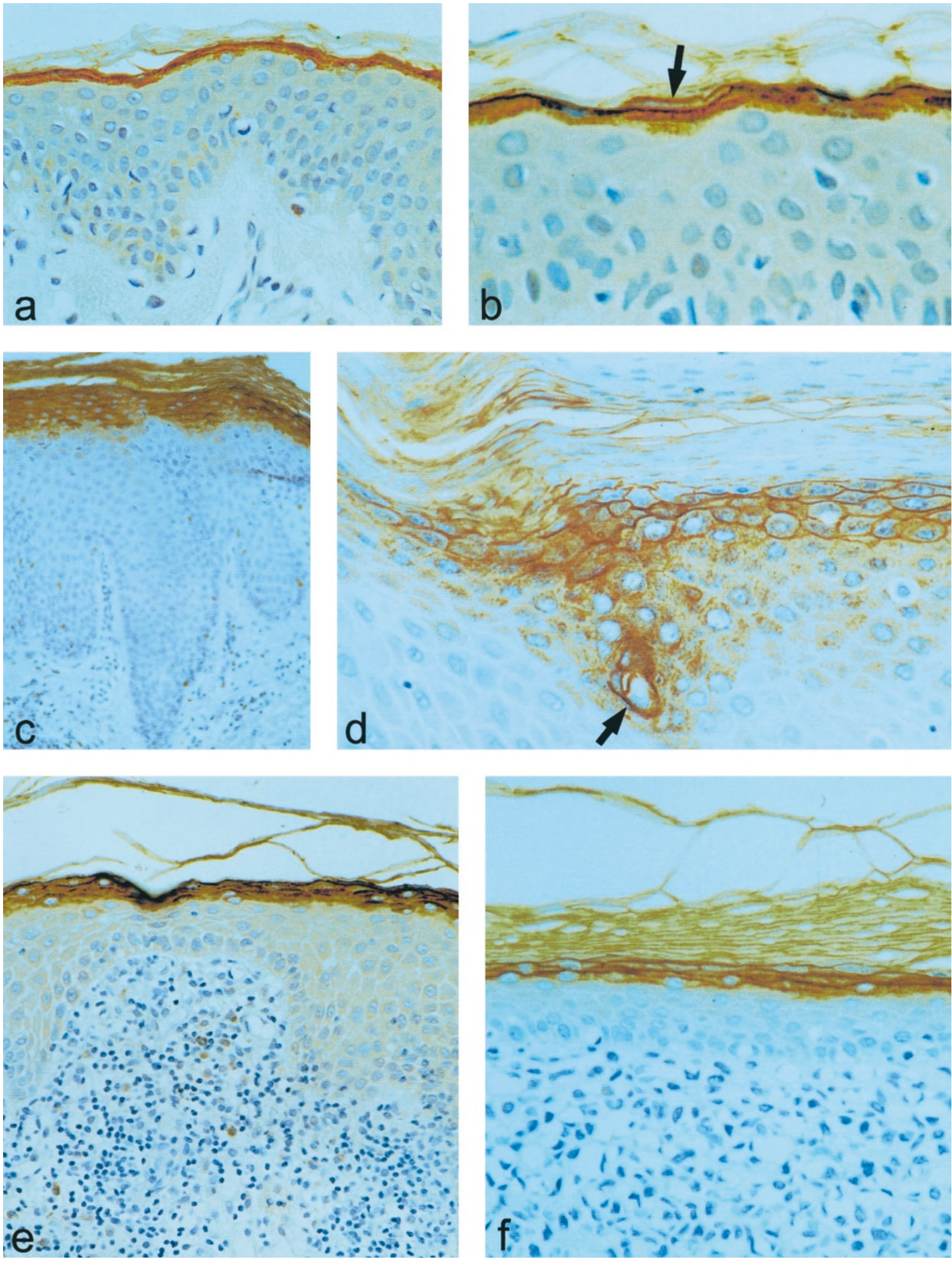

\section{Figure 2.}

a and b, Immunoreactivity of Cdsn in paraffin-embedded normal skin using the monoclonal antibody G36-19 in a peroxidase-antiperoxidase method shows Cdsn expression in the stratum granulosum confined to a compact layer of superficial granular cells. Labeling is predominantly cytoplasmic. Cdsn immunoreactivity is retained throughout the full thickness of the stratum corneum. Occasional flattened nucleated cells in the basal layer of the stratum corneum $(\rightarrow)$ show a cell surface immunoreactivity pattern (b). c, G36-19 immunoreactivity of paraffin-embedded psoriatic skin shows that Cdsn expression is strong and present in multiple layers of the epidermis. $d$, Higher magnification demonstrates that the expression pattern varies from granular cytoplasmic immunoreactivity to cell surface expression in the more superficial layers of the stratum spinosum. Acrosyringium is also immunoreactive for Cdsn $(\rightarrow)$. In lichen planus (e) and mycosis fungoides (f), immunoreactivity with the G36-19 antibody shows that Cdsn is expressed in a compact and discrete layer of cells at the upper margin of the stratum granulosum, similar to that observed for normal skin. The Cdsn immunoreactivity pattern is predominantly cytoplasmic, although some cells show cell surface labeling. 
from stratum spinosum to stratum corneum (Fig. 2, c and d). Expression of Cdsn in lesional psoriasis varied from dense, granular, cytoplasmic immunoreactivity in lower and middle layers of the stratum spinosum to cell surface and weak cytoplasmic expression in upper stratum spinosum, with cell surface expression in the stratum corneum. As with normal skin, acrosyringia in psoriatic epidermis were immunoreactive for Cdsn. Biopsies taken from clinically normal, nonlesional skin in front of the advancing psoriatic lesion showed the same pattern of immunoreactivity for Cdsn as normal skin.

Other Skin Diseases. In chronic atopic dermatitis, lichen planus, mycosis fungoides, and pityriasis rubra pilaris, Cdsn was expressed uniformly in a layer of the epidermis, up to three cells thick in the upper stratum granulosum and throughout the entire stratum corneum (Fig. 2, e and f). This immunoreactivity was predominantly cytoplasmic, although occasional cells in the most superficial stratum granulosum showed cell surface immunoreactivity. There was little variation in the pattern of Cdsn immunoreactivity observed between the different inflammatory conditions. Although representing a greater area of epidermis expressing Cdsn than was seen in normal skin, the extent of Cdsn expression in these conditions never matched the immunoreactivity seen in psoriasis and did not extend into the stratum spinosum.

\section{Immunoelectron Microscopy}

Transmission electron microscopy clearly confirmed that, in both normal and psoriatic epidermis, the major location of Cdsn on the cell surface is within desmosomes (Figs. 3 and 4). In both psoriasis and normal skin, intracellular immunoreactivity of Cdsn was also observed within cytoplasmic vesicles. Release of Cdsn into the extracellular space in normal skin was restricted to the most superficial granular cells (Fig. 3), whereas in psoriasis, Cdsn release occurred three to four cell layers below the cornified cell layer (Fig. 3). Psoriatic epidermis was immunoreactive to the involucrin antibody (Fig. 4), which confirmed earlier observations (Ishida-Yamamoto et al, 1995) that formation of the cornified cell envelope begins at a much lower level of the epidermis than is observed in normal skin. Extracellular release of Cdsn and initiation of involucrin association with the cell envelope are almost simultaneous events, but Cdsn release seemed to be complete before full maturation of the cornified cell envelope. Obvious differences were not observed in desmosomal structure nor were quantitative differences found in the amount of label present or in the intracellular distribution of Cdsn in psoriatic versus normal skin.

\section{Discussion}

In this study, we compared expression patterns of the epidermal glycoprotein Cdsn in normal skin and psoriasis at both the light and electron microscopic levels. These observations were compared with Cdsn expression in a range of other skin conditions.
Both current and previously published results (Haftek et al 1997; Mils et al 1992; Serre et al 1991) suggest that in normal skin, synthesis and release of Cdsn is tightly controlled, with Cdsn synthesis occurring in the most superficial cells of the stratum granulosum and release of Cdsn restricted to the interface between stratum granulosum and stratum corneum. In psoriatic skin, contrasting with both normal skin and inflammatory skin conditions, such restricted control of Cdsn synthesis seems to be diminished, with widespread expression of Cdsn in multiple layers of stratum spinosum, and both cytoplasmic and cell surface expression. In particular, we observed a significant increase in Cdsn expression in lesional psoriasis compared with both nonlesional skin and normal control skin. In chronic atopic dermatitis, lichen planus, mycosis fungoides, and pityriasis rubra pilaris, control of Cdsn expression is more similar to the expression observed in normal epidermis, although Cdsn synthesis occurs in a slightly broader band of superficial granular cells in these conditions. Similar to normal skin, few cells in the stratum granulosum exhibit cell surface immunoreactivity.

These findings are paralleled by immunoelectron microscopic observations. In normal skin, Cdsn labeling of desmosomes in living layers was limited to a narrow zone of cells at the interface between the stratum granulosum and stratum corneum, whereas in psoriasis, Cdsn labeling of desmosomes was visible in multiple cell layers within the epidermis. Double labeling results using antibodies to Cdsn and involucrin were consistent with earlier observations that in psoriasis the cornified cell envelope begins to form at a lower level of the epidermis than in normal skin (Ishida-Yamamoto and lizuka, 1995). Furthermore, we demonstrated that extracellular release of Cdsn is complete before completion of incorporation of involucrin into the cell envelope.

These findings suggest that, in psoriasis, keratinocytes may have a mechanism for accelerating Cdsn release. Psoriatic epidermis differs from normal and other diseased epidermis in that the stratum granulosum is missing and the formation of the cornified cell envelope begins at a much lower level of the epidermis (Ishida-Yamamoto and lizuka, 1995), indicated by the premature incorporation of its major constituent, involucrin, into the cornified cell envelope. Other markers of differentiation, including membrane-bound transglutaminase (Bernard et al, 1988) and enzymes thought to be involved in epidermal desquamation (Ekholm and Egelrud, 1999), are also expressed earlier in psoriatic epidermis than in normal skin. Thus, the pattern of Cdsn expression seen in psoriasis may be the result of a more generalized dysfunction in keratinocyte differentiation, which may be related to the increased levels of intracellular calcium and increased protein kinase $\mathrm{C}$ activation (Ishida-Yamamoto and lizuka, 1995), in psoriatic epidermis. Thus, in psoriasis, factors responsible for the premature incorporation of involucrin into the cell envelope may also influence the synthesis and/or secretion of Cdsn, resulting in the 


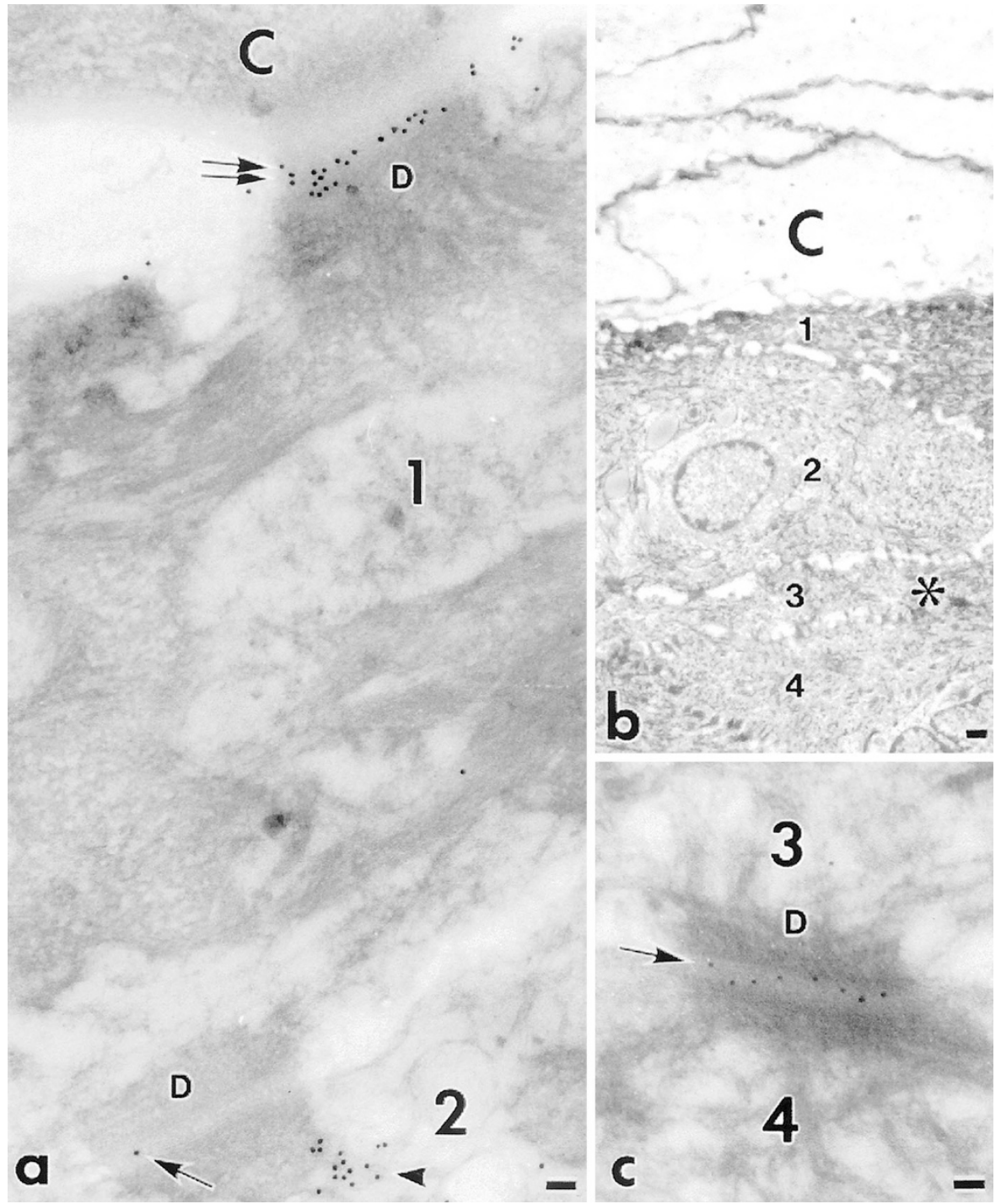

Figure 3.

Cdsn immunoelectron microscopy labeling of normal (a) and psoriatic skin (b and c) using gold particle-conjugated antibodies. a, In normal skin, Cdsn labeling occurs mainly at the interface between the first stratum corneum cell $(C)$ and the uppermost granular layer (1). Cdsn (arrows) is localized at the desmosome $(D)$. There is occasional Cdsn labeling on desmosomes between cell layers 1 and 2 of the granular layer. However, there is no extracellular Cdsn labeling in the other cell layers, although intracellular labeling is present (arrowhead). b. Psoriatic epidermis showing stratum corneum $(C)$ and four cell layers immediately beneath the stratum corneum ( 1 to 4). The position of a desmosome between cells 3 and 4 is marked by an asterisk and is shown at higher magnification in c. c, In psoriatic skin, in contrast to normal skin, extracellular labeling of desmosomes (D) for Cdsn (arrow) is present in the third and fourth layer of cells beneath the stratum corneum. Scale bars: $0.1 \mu \mathrm{m}$ in a and $\mathrm{c}, 1 \mu \mathrm{m}$ in $\mathrm{b}$.

completion of extracellular release of Cdsn before formation of the mature cornified cell envelope. Alternatively, variant forms of Cdsn, coded for by particular alleles of the Cdsn gene, may have an enhanced ability for translocation to and extracellular release from the cell surface and may affect incorporation of involucrin into the cell envelope.

However, when immunoreactivity patterns were compared between psoriatic individuals genotyped for Cdsn gene polymorphisms, we found no relationship between genotype and Cdsn immunoreactivity pattern (data not shown). There is no firm evidence that these allelic variants lead to differences in functioning of the Cdsn molecule, although the three polymorphisms investigated (at nucleotides +619 , +1240 , and +1243 ) lead to changes in the amino acid sequence of Cdsn (Ishihara et al, 1996), and thus may have functional importance. The G36-19 and F28-27 antibodies recognize epitopes in the central domain of the Cdsn molecule that are conserved during the proteolytic digestion that occurs in the stratum corneum (Simon et al, 1997). The serine- and glycine-rich terminal domains of Cdsn, which are likely to exist as adhesive loops and are cleaved before desquamation, are not recognized by these antibodies. Therefore, we may not have identified any differences in the Cdsn molecule related to different alleles of the gene because these molecular differences do not affect expression of the epitopes recognized by the antibodies used. To undertake a more complete expression mapping study would require the use of antibodies specific for epitopes across the whole molecule. 

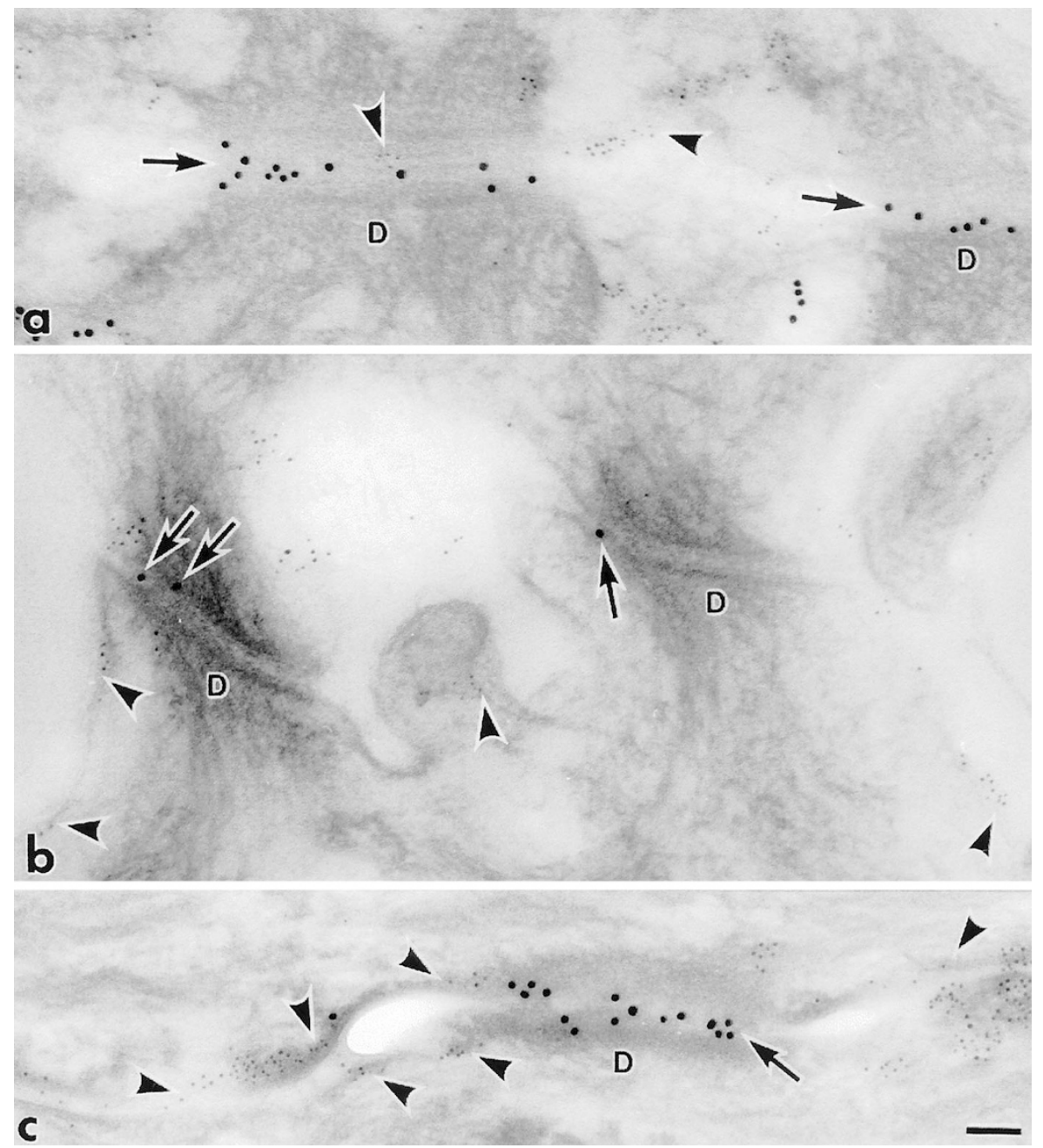

\section{Figure 4.}

Double immunoreactivity for Cdsn (10-nm gold) and involucrin (5-nm gold) in normal (a) and psoriatic skin (b and c). a, In normal skin, desmosomes (D) on cells at the interface between the stratum granulosum and stratum corneum demonstrate colocalization of Cdsn (arrows) (10-nm gold) and involucrin (arrowheads) (5-nm gold). Cell membranes show involucrin labeling (arrowheads) consistent with initiation of cell envelope assembly. b, In psoriatic epidermis, keratinocytes within the stratum spinosum where Cdsn (arrows) is beginning to be incorporated into desmosomes also show labeling of cell membranes for involucrin (arrowheads). Neither Cdsn nor involucrin labeling is seen in equivalent cell layers in normal skin. These findings in psoriatic epidermis are consistent with earlier release of Cdsn and earlier cell envelope formation than seen in normal skin. c, In mature cornified cell envelopes from the superficial epidermis of psoriatic skin, desmosomes ( $D$ ) show increased labeling for Cdsn (arrow) compared with those desmosomes at lower levels in the epidermis (shown in b). This suggests that extracellular Cdsn release is complete (arrow) although cell envelope-associated involucrin immunolabeling (arrowheads) implies that the cell envelope is not fully mature. Scale bar: $0.1 \mu \mathrm{m}$.

The role of Cdsn in the biology of the epidermis has yet to be fully determined. However, our results indicate that Cdsn is expressed differently in psoriasis compared with normal skin and other skin conditions, and may indicate that the molecule is processed differently in psoriatic epidermis, providing further evidence for a particular role for Cdsn in psoriasis. Whether these differences in Cdsn expression result from changes already occurring in psoriatic skin or are fundamental effects contributing to the pathogenesis of the disease is still to be determined. Psoriasis is a complex multifactorial disease, which implies that functional polymorphisms are more likely to be relevant to the genetic basis of the disease rather than gene mutations. Our data are consistent with a role for Cdsn in psoriasis and further support Cdsn as a candidate psoriasis-susceptibility gene. However, a combination of further genetic and functional studies of Cdsn are required to more clearly define the role of Cdsn in psoriasis and epidermal cell biology.

\section{Materials and Methods}

\section{Immunohistochemistry}

Following ethical approval and informed consent, paired biopsies were taken from lesional skin and clinically normal, nonlesional skin, $1 \mathrm{~cm}$ in advance of the active edge of the lesion, as identified by laser Doppler fluxmetry (Davison et al, 2001) in adult psoriatic patients $(n=17)$. Biopsies were also taken from normal volunteers $(n=6)$. Tissue samples were snapfrozen in isopentane in liquid nitrogen and stored at $-80^{\circ} \mathrm{C}$. Archival skin biopsies from a range of skin pathologies were obtained from the St John's Institute of Dermatology histopathology laboratory (King's Col- 
lege, London, United Kingdom). Formalin-fixed paraffin-embedded sections were obtained from the following conditions: psoriasis $(n=6)$, chronic atopic dermatitis $(n=3)$, lichen planus $(n=3)$, mycosis fungoides $(n=3)$, pityriasis rubra pilaris $(n=2)$, and normal skin $(n=3)$.

Immunohistochemistry was performed using two monoclonal antibodies to Cdsn, G36-19 and F28-27 (Serre et al, 1991), which label different epitopes in the central domain of the Cdsn protein (Guerrin et al, 1998). Five-micron-thick sections were mounted on glass slides and either fixed in acetone (frozen tissue) or dewaxed in xylene and alcohol and microwave pretreated (paraffin-embedded tissue). Sections were incubated with primary antibody at a concentration of $10 \mathrm{ng} / \mu \mathrm{l}$ (1:50 dilution) for 60 minutes in a standard peroxidase anti-peroxidase method and visualized with diaminobenzidine. On control sections, primary antibody was either omitted or replaced with a primary antibody of irrelevant specificity. Immunoreactivity was performed on both frozen and paraffin-embedded tissue, enabling us to use a large quantity of archival material of unequivocal diagnosis and to ensure that the paraffin-embedding process did not adversely affect the antibody binding seen on frozen tissue (or vice versa).

\section{Quantification}

The numbers of living cell (ie, nucleated cell) layers in the epidermis expressing Cdsn were counted in all samples of normal skin, nonlesional skin, and psoriasis. Differences in these counts were statistically analyzed using a two-sample $t$ test.

\section{Immunoelectron Microscopy}

For immunoelectron microscopy, tissue was prepared using a freeze substitution method and immunolabeled using a post-embedding technique (IshidaYamamoto et al, 1996). Briefly, tissue was cryoprotected in $15 \%$ glycerol in phosphate buffered saline, then rapidly frozen in liquid propane, subjected to cryo-substitution in methanol, and embedded in Lowicryl K11M resin (Chemische Werke Lowi, Waldkraiburg, Germany). Ultrathin sections were cut and labeled with antibodies to Cdsn and involucrin (BT601; Biomedical Technologies, Stoughton, Massachusetts). Secondary labeling with gold-conjugated secondary antibodies (10-nm gold for Cdsn and 5-nm gold for involucrin) was then performed. Control sections were incubated with normal serum in place of primary antibody. Sections were stained with uranyl acetate for improved contrast.

\section{References}

Allen $\mathrm{MH}$, Veal C, Faassen A, Powis SH, Vaughan RW, Trembath RC, and Barker JNWN (1999). A non-HLA gene within the MHC in psoriasis. Lancet 353:1589-1590.

Balendran N, Clough RL, Arguello JR, Barber R, Veal C, Jones AB, Rosbotham JL, Little A-M, Madrigal A, Barker JNWN, Powis SH, and Trembath RC (1999). Characterization of the major susceptibility region for psoriasis at chromosome 6p21.3. J Invest Dermatol 113:322-328.

Barker JNWN (1991). Pathophysiology of psoriasis. Lancet 338:227-230.

Bernard BA, Asselineau D, Schaffer-Deshayes L, and Darmon MY (1988). Abnormal sequence of expression of differentiation markers in psoriatic epidermis: Inversion of two steps in the differentiation program. J Invest Dermatol 90: 801-805.

Burden $A D$, Javed S, Bailey $M$, Hodgins $M$, Connor $M$, and Tillman D (1998). Genetics of psoriasis: Paternal inheritance and a locus on chromosome 6p. J Invest Dermatol 110:958960.

Camp RDR (1998). Psoriasis. In: Champion RH, Burton JL, Burns DA, and Breathnach SM, editors. Textbook of dermatology. Oxford: Blackwell Science, 1589-1649.

Davison SC, Ballsdon A, Allen MH, and Barker JNWN (In press, 2001). Early migration of cutaneous lymphocyte associated antigen (CLA) positive cells into evolving psoriatic plaques. Exp Dermatol.

Ekholm E and Egelrud T (1999). Stratum corneum chymotryptic enzyme in psoriasis. Arch Dermatol Res 291:195-200.

Elder JT, Nair RP, Guo S-W, Henseler T, Christophers E, and Voorhees JJ (1994). The genetics of psoriasis. Arch Dermatol 130:216-224.

Guerrin M, Simon M, Montezin M, Haftek M, Vincent C, and Serre G (1998). Expression cloning of corneodesmosin proves its identity with the product of the $S$ gene and allows improved characterization of its processing during keratinocyte differentiation. J Biol Chem 273:22640-22674.

Haftek M, Simon M, Kanitakis J, Maréchal S, Claudy A, Serre G, and Schmidt D (1997). Expression of corneodesmosin in the granular layer and stratum corneum of normal and diseased epidermis. Br J Dermatol 137:864-871.

Ishida-Yamamoto A, Eady RA, Watt FM, Roop DR, Hohl D, and lizuka $\mathrm{H}$ (1996). Immunoelectron microscopic analysis of cornified cell envelope formation in normal and psoriatic epidermis. J Histochem Cytochem 44:167-175.

Ishida-Yamamoto A and lizuka H (1995). Differences in involucrin immunolabelling within cornified cell envelopes in normal and psoriatic epidermis. J Invest Dermatol 104:391395.

Ishihara M, Yamagata N, Ohno S, Naruse T, Ando H, Kawata $\mathrm{H}$, Ozawa A, Ohkido N, Mizuki T, Shiina H, Ando H, and Inoko H (1996). Genetic polymorphisms in the keratin-like S gene within the major histocompatibility complex and association analysis on the susceptibility to psoriasis vulgaris. Tissue Antigens 48:182-186.

Jenisch S, Koch S, Henseler T, Nair RP, Elder JT, Watts CE, Westphal E, Voorhees JJ, Christophers E, and Kronke M (1999). Corneodesmosin gene polymorphism demonstrates strong linkage with HLA and association with psoriasis vulgaris. Tissue Antigens 54:439-449.

Mils V, Vincent C, Croute F, and Serre G (1992). The expression of desmosomal and corneodesmosomal antigens shows specific variations during the terminal differentiation of epidermis and hair follicle epithelia. J Histochem Cytochem 40:1329-1337. 
Nair RP, Henseler T, Jenisch S, Stuart P, Bichakjian CK, Lenk W, Westphal E, Guo SW, Christophers E, Voorhees JJ, and Elder JT (1997). Evidence for two psoriasis susceptibility loci (HLA and 17q) and two novel candidate regions (16q and 20 p) by genome-wide scan. Hum Mol Genet 6:1349-1356.

Nair RP, Stuart P, Henseler T, Jenisch S, Chia NVC, Westphal E, Schork NJ, Kim J, Lim HW, Christophers E, Voorhees JJ, and Elder JT (2000). Localization of psoriasis-susceptibility locus PSORS1 to a $60-\mathrm{kb}$ interval telomeric to HLA-C. Am J Hum Genet 66:1833-1844.

Nickoloff BJ and Wrone-Smith T (1999). Injection of prepsoriatic skin with CD4+ cells induces psoriasis. Am J Pathol 155:145-158.

Oka A, Tamiya G, Tomizawa M, Ota M, Katsuyama Y, Makino S, Shiina $T$, Yoshitome M, lizuka M, Sasao $Y$, Iwashita $K$, Kawakubo $Y$, Sugai J, Ozawa A, Ohkido M, Kimura M, Bahram S, and Inoko H (1999). Association analysis using refined microsatellite markers localizes a susceptibility locus for psoriasis vulgaris within a $111 \mathrm{~kb}$ segment telomeric to the HLA-C gene. Hum Mol Genet 8:2165-2170.

Serre G, Mils V, Haftek M, Vincent C, Croute F, Reano A, Ouhayoun J-P, Bettinger S, and Soleilhavoup J-P (1991). Identification of late differentiation antigens of human epithelia, expressed in reorganized desmosomes and bound to cross-linked envelope. J Invest Dermatol 97:1061-1072.
Simon M, Montezin M, Guerin M, Durieux J-J, and Serre G (1997). Characterization and purification of human corneodesmosin, an epidermal basic glycoprotein associated with corneocyte-specific modified desmosomes. J Biol Chem 272:31770-31776.

Tazi Ahnini R, Camp NJ, Cork NJ, Mee JB, Keohane SG, Duff GW, and di Giovine FS (1999). Novel genetic association between the corneodesmosin (MHC S) gene and susceptibility to psoriasis. Hum Mol Genet 8:1135-1140.

Trembath RC, Clough RL, Rosbotham JL, Jones AB, Camp RDR, Frodsham A, Browne J, Barber R, Terwilliger J, Lathrop GM, and Barker JNWN (1997). Identification of a major susceptibility locus on chromosome $6 p$ and evidence of further disease loci revealed by a two stage genome-wide search in psoriasis. Hum Mol Genet 6:813-820.

Zhou Y and Chaplin DD (1993). Identification in the HLA class I region of a gene expressed late in keratinocyte differentiation. Proc Natl Acad Sci USA 90:9470-9474. 